\title{
DEMAND FORECAST OF SEMICONDUCTOR PRODUCTS BASED ON TECHNOLOGY DIFFUSION
}

\author{
Chen-Fu Chien, Yun-Ju Chen
}

Department of Industrial Engineering \& Engineering Management

National Tsing Hua University

Hsinchu 30013, TAIWAN, ROC

\author{
Jin-Tang Peng \\ Department of Business Administration \\ Yuanpei University \\ Hsinchu 30015, TAIWAN, ROC
}

\begin{abstract}
Demand forecast plays a critical role to determine capital investment for capacity planning. Given the involved uncertainties and long lead-time for capacity expansion, semiconductor companies have to predict future demands as a basis for related manufacturing strategic decisions. As semiconductor products in a consumer era become more diversified with shortening life cycle, demand forecast also becomes more complex and difficult. This study aims to develop a demand forecast model based on product life cycle and technology diffusion. While little research has been done to employ diffusion models to forecast the demands of semiconductor products. The proposed model modifies a multi-generation diffusion model incorporating marketing variables into the model for semiconductor product and uses nonlinear least square method to estimate the parameters. An empirical study is conducted to validate the proposed model with real data of semiconductor products. This research concludes with discussion on future research directions.
\end{abstract}

\section{INTRODUCTION}

In order to utilize the resources effectively, semiconductor companies need to make manufacturing strategic decisions and associated operations including capacity planning, production planning, material requirement planning, inventory control, and scheduling (Wu and Chien 2008). Demand forecast plays a critical role to determine capital investment for capacity planning to improve capacity utilization and capital effectiveness. However, given the involved uncertainties and long lead time for capacity expansion, semiconductor companies have to predict future demands as a basis for related manufacturing strategic decisions under the risks of capacity shortage and/or surplus along the time.

Moore's Law has driven the semiconductor industry to keep technology migrations and wafer enlargement to maintain the cost reduction per transistor and the growth of semiconductor industry (Leachman, Ding, and Chien
2007). In other words, Moore's Law shortens the technology product life cycle and products substitution time. On the other hand, consumption of diversified semiconductor products is affected by various economics factors, which lead to demand uncertainty and increasing risks of capacity shortage and/or surplus in the demand forecast. As semiconductor products in a consumer era become more diversified with shortening life cycle, demand forecast also becomes more complex and difficult.

Most of all companies forecast demand in the future by using market information and their related experience or domain knowledge. They deal with the problem subjectively. The result of this method might have low accuracy and large errors. Additionally, it needs to repeat the process with their market information, related experience or domain knowledge for different products. Thus, it is necessary to construct a suitable and general model to forecast demand precisely.

Different forecasting methods have been applied in different areas. Most of existing demand forecasting studies employed the time series methods. But these methods are difficult to express the entire adoption process of the new products. Therefore, it is necessary to construct a model for express the entire adoption process. It can help managers to make well decisions for the new product. Inherent forecasting methods are mainly designed for a single generation without considering the substitution between generations and therefore they needs to execute the forecasting repetitively. However, the characteristics of semiconductor products are multi-generation under a segment. A segment is manufactured under different technologies. For this reason, it is crucial to forecast a semiconductor product under different generations one time. Multi-generation diffusion models can deal with the problem for the characteristics of semiconductor products. On the other hand, diffusion models are applied to consumer durables primarily. Little research has been done to employ diffusion models to forecast the demands of semiconductor products. It is crucial to construct a multigeneration diffusion model for semiconductor products. 
Chien, Chen, and Peng

This study aims to construct a diffusion model for forecasting semiconductor products based on historical data. Demand forecast is a fundamental of production decisions and production planning. Manufacturing strategic decisions involve the tradeoffs among productivity, quality, delivery, and cost (Chien, Wang, and Wang 2007). The precision of demand forecast may decrease inventory cost, promote the quality of customer service and increase market competition. This study develops a product life cycle based on the proposed diffusion model and employs it to forecast demand, in which nonlinear least square method is employed to estimate the parameters.

\section{DEMAND FORECAST FRAMEWORK}

This study proposes a demand forecast framework for semiconductor products in order to predict the demand and plan the capacity as shown in Figure 1. The framework consists of six phases corresponding to the six subsections.

The terminology and notations used in this study are as follows:

$F_{i}(t) \quad-$ the cumulative density function at time $\mathrm{t}$ for generation $\mathrm{i}$

$f_{i}(t) \quad$ - the probability density function at time $\mathrm{t}$ for generation $\mathrm{i}$

$s_{i}(t) \quad-$ the actual sales of products at time $t$ for generation $\mathrm{i}$

$X_{i}(t) \quad$ - the cumulative market effects

$\hat{s}_{i}(t) \quad-$ the estimated sales of products at time $\mathrm{t}$ for generation $\mathrm{i}$

$\tau_{i} \quad$ - the introduction time for generation $\mathrm{i}, \tau_{\mathrm{i}} \geq 1$

$p_{i} \quad$ - the proportion of mass media communication for generation $\mathrm{i}$

$q_{i} \quad$ - the proportion of word of mouth for generation $\mathrm{i}$

$m_{i} \quad$ - the market potential (incremental market potential) for generation $\mathrm{i}$

$\rho_{i} \quad$ - $\quad$ the average repeat purchase rate for generation $\mathrm{i}$

$M_{i} \quad$ - Total market potential for generation i, $M_{i}=m_{i} \cdot \rho_{i}$

$\alpha_{t} \quad$ - the seasonal factor at time $\mathrm{t}$

$\beta \quad \quad \quad$ the effectiveness of the price

$p r_{i}(t) \quad$ - the price at time $\mathrm{t}$ for generation $\mathrm{i}$

$g_{t} \quad \quad \quad$ - the growth rate at time $\mathrm{t}$

$n \quad$ - the number of generations

$l \quad$ - the number of periods

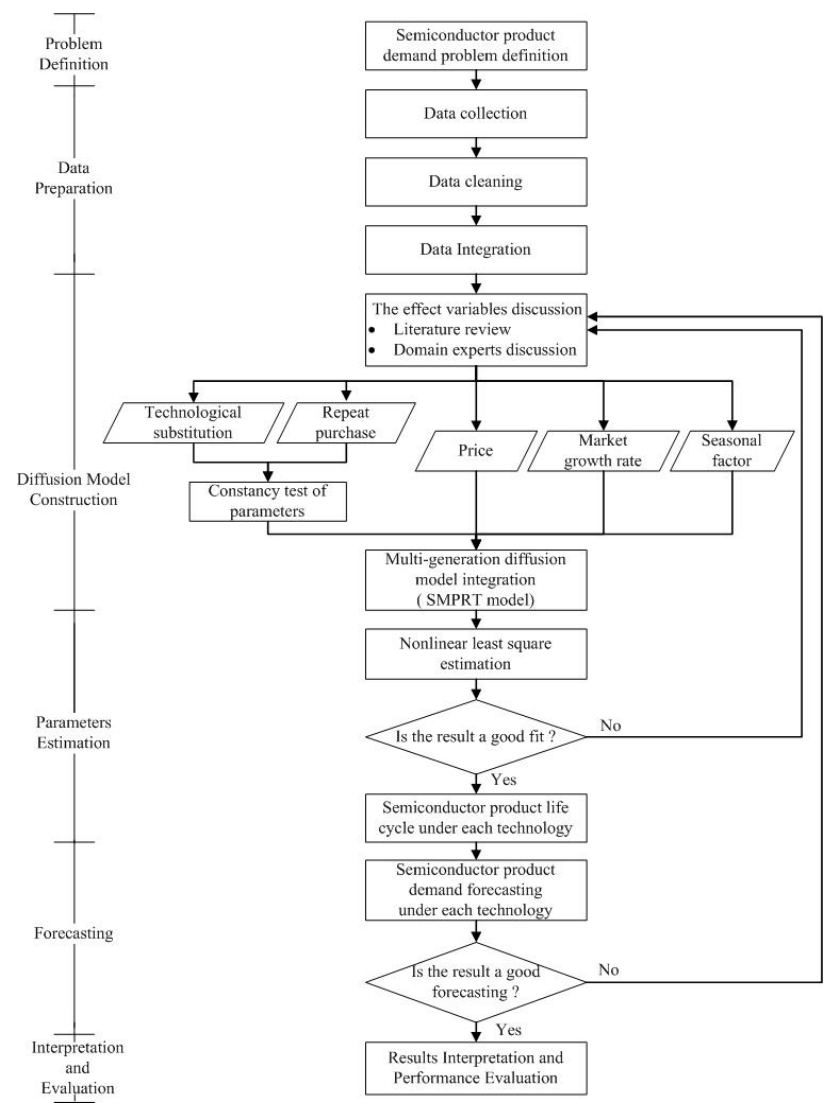

Figure 1: Research framework

\subsection{Problem Definition}

The orders in the semiconductor industry are customized and make-to-order. For the moment, the developments of the semiconductor manufacturing technologies face to shorten the line-width and expand the diameter of the wafer in order to provide better products with more applications and functions to the markets. Semiconductor industry grows rapidly that shorten the product life cycle (PLC) of semiconductor products. PLC is important information to plan market strategies. In semiconductor industry, R\&D costs and capital investments are a huge amount of money. In order to make to allocate the resource efficiently and well planning, it is a critical issue to describe the PLC and forecast the demand in the future. But the process of forecasting demand in semiconductor industry is difficult for uncertainty and risk. In addition, it is hard to forecasting the medium and long-term only for historical data of old technologies because of the technological migration and demand variation.

How to plan the capacity is an important decision for companies. However, capacity planning relies on demand forecasting. If demand forecasting values are overestimated than the actual values unfolded later, capacity can- 


\section{Chien, Chen, and Peng}

not be utilized efficiently. It might lead the idle capacity and waste. When demand forecasting values are underestimated than actual values unfolded in the future, capacity will not be sufficient to fulfill the demands then. It might lose the orders or customers. In order to predict product demand precision, this study will construct a demand forecast framework to forecast product demand in the future and assist the manager to decision the capacity planning. Furthermore, the demand forecast framework could be applied to support capacity allocation and capacity expansion decisions.

\subsection{Data Preparation}

The marketing sales department records and stores each transaction record in the database. The collected data often includes noisy, missing and inconsistent data. It is necessary to assure the quality of data in order to accelerate the effectiveness of the model. Hence, data preparation can be used improve the quality of the data and construct the effective demand forecasting model. The data preparation process includes data collection, data cleaning, and data integration.

Collect the requisite data for this study from the database. To improve the quality of data, it is necessary to deal with redundant data, inconsistent data, missing data, and so on. Missing data can be deleted or replaced by some data cleaning methods in the data cleaning process. The data cleaning of this study needs to delete the some rows and columns that are redundant in this study.

Data integration merges the data of different sources

Table 1: Product demand Integration

\begin{tabular}{|c|c|c|c|c|}
\hline Customer & Product & Technology & $200 \times 1 Q$ & $200 \times 2 Q$ \\
\hline 1 & $\mathrm{X}$ & A & 50 & 30 \\
\hline 2 & X & B & 25 & 30 \\
\hline 1 & $\mathrm{X}$ & $\mathrm{C}$ & 50 & 80 \\
\hline 2 & $\mathrm{X}$ & $\mathrm{C}$ & 70 & 90 \\
\hline 2 & Y & B & 30 & 50 \\
\hline 3 & $\mathrm{X}$ & D & 70 & 105 \\
\hline 3 & $X$ & B & 58 & 70 \\
\hline 1 & X & D & 80 & 100 \\
\hline 2 & Y & $\mathrm{C}$ & 60 & 78 \\
\hline \multicolumn{5}{|c|}{ 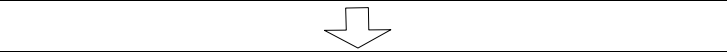 } \\
\hline Product & \multicolumn{2}{|c|}{ Technology } & $00 \times 1 Q$ & $200 \times 2 Q$ \\
\hline $\mathrm{X}$ & \multicolumn{2}{|c|}{$\mathrm{A}$} & 50 & 30 \\
\hline $\mathrm{X}$ & \multicolumn{2}{|c|}{ B } & 83 & 100 \\
\hline $\mathrm{X}$ & \multicolumn{2}{|c|}{$\mathrm{C}$} & 120 & 170 \\
\hline $\mathrm{X}$ & \multicolumn{2}{|c|}{ D } & 150 & 200 \\
\hline Y & \multicolumn{2}{|c|}{ B } & 30 & 50 \\
\hline Y & \multicolumn{2}{|c|}{$\mathrm{C}$} & 60 & 78 \\
\hline
\end{tabular}

into a file for this study. For example, the price of the products, the growth rate of semiconductor industry, and the demand of the products need to merge in the same file in order to analyze the data conveniently. The data need to be putted in suitable segments by quarters and different technologies, respectively. Additionally, it is necessary to sum up the same technology and the same product data such as Table 1. The data also need to transform to suitable formats for this study.

\subsection{Diffusion Model Construction}

This section aims to develop a diffusion model for semiconductor products. This study constructs a multigeneration diffusion model based on Norton and Bass model. Firstly, test the parameters whether they changes across generations. Based on the result of statistical hypotheses, determine the parameters whether they change across generations. In the semiconductor industry, some characteristics affect the demand including the price effect, the growth rate of semiconductor industry, and so on. The proposed diffusion model in this study matches up the characteristics of the semiconductor product to attain the valuable information and therefore it incorporates the technological substitution, repeat purchases, price effect, market growth rate and seasonal factor into the model.

\subsubsection{The Effect Variables}

There are five factors considered in this study including the technological substitution, repeat purchases, price, market growth rate and seasonal factor. The factors are selected through literature review, judgments from domain experts or statistical methodologies.

1. Technological substitution effects

As the result of Moore's law, semiconductor industry develops rapidly. Newer technologies continually introduce to the market. Newer technologies are always superior due to newer technologies with attached applications and functions, hence newer technologies will replace older ones step by step. The introduction of newer technology also widens the market potential and affects the buyers of the older technology to buy the newer technology to substitute the older ones. In other words, the sales of the older generation products might be substituted for the newer generation products. Most forecasting methods only focus on the new product itself and ignore the older generation products that might compete with new products.

The single generation diffusion models or other forecasting methods are not suitable for semiconductor products. It is essentially technological innovation for the new products of semiconductor industry. It needs to consider both diffusion and substitution for semiconductor products in this study and therefore multigeneration diffusion models are feasible for semiconductor products.

2. Repeat purchases 
Consider repeat purchase in this thesis, because Adopters don't purchase only one unit. Both Norton and Bass (1987) and Bass and Bass (2001) consider repeat purchase in their model. Bass and Bass (2001) revised mainly the multigeneration model proposed by Norton and Bass. The model divided the adopters into two parts: first adopters and repeated adopters respectively. In the Norton and Bass model, it considered that the average purchase rate is a constant $\rho_{i}$ and therefore $M_{i}=\rho_{i} \cdot m_{i} \cdot M_{i}$ can be estimated, but they didn't identify $\rho_{i}$ and $m_{i}$ respectively.

3. Price effects

Price is a pivot factor to affect customers what to buy and therefore the price effect of multigeneration products is important. The pricing strategy considers not only the stages of the product life cycle but also the substitution of newer generation products. In general, the demand curve shows as the exponential curve. The higher is the price of the products, and the smaller is the quantity of the demand. On the other hand, the price of product is down, the demand might be up. As the newer generation products introduce to the market, the price of the newer generation products will affect customers to decide what to buy. If the expected future price will be increased or reduced, the behaviors of the customers also change. For semiconductor products, the price goes down quickly in the initial stage of the products and varies steady in the latter stage.

Bass, Krishnan, and Jain (1994) incorporated the marketing effect into the Bass model for a single generation. The marketing effect contained price and advertising expenditures in this model. The model has been applied to consumer durables such as the color television, room air conditioner and so on. This approach extends this model to multi-generation model for semiconductor products.

4. Market growth rates

Growth rate is a factor to describe the social environment. The variation of customer behaviors is affected by the growth rate. When the growth rate is rise, the demand might be up.

5. Seasonal factors

In general, many business activities will be affected by seasonal factors. Semiconductor products applied to various industries such as PC manufacturing, communication industry, and so on. However, the sales of PC may be affected by seasonal factors. For example, the sales of PC will increase at Christmas.
It needs to consider the effect of seasonality for neglecting the effect of seasonality may lead to overestimate/underestimate demand.

\subsubsection{The Proposed Model (SMPRT model)}

Most multi-generation diffusion models only considered the basic adoption process and therefore didn't incorporate marketing effects into the models. We extend the basic models to incorporate the seasonal factor, market growth rate, price and technological substitution effect into the proposed model. The proposed model is called SMPRT model (seasonal factor, market growth rate, price, repeat purchases and technological substitution).

The products with different generations are same products for different technologies and therefore the diffusion process of the products is similar. Norton and Bass (1987) assumed that the internal and external coefficients are constant across different generations in order to reducing the complexity of the parameters. On the other hand, Islam and Meade (1997) released the assumption, and test the constancy of parameters.

Following, we state the steps in SMPRT model.

Step1. Compare Norton and Bass (NB) and Islam and Meade (IM) model

The models are expressed as follows.

$s_{i}(t)=f_{i}(t)\left[M_{i}+f_{i-1}(t)\left[M_{i-1}+f_{i-2}(t)\left[M_{i-2}+\cdots f_{2}(t)\left[M_{2}+f_{1}(t) M_{1}\right] \cdots\right]\right]\right]\left[1-f_{i+1}(t)\right]$

The difference between NB and IM model is the parameters whether they changes across generations or not.

- Norton and Bass model

In this model, the parameters are constant across generations, and therefore $\mathrm{p}=\mathrm{p}_{\mathrm{i}}$ and $\mathrm{q}=\mathrm{q}_{\mathrm{i}}$ for all $\mathrm{i}$.

$$
F_{i}(t)=\frac{\left[1-e^{-\left(p_{i}+q_{i}\right)\left(t-\tau_{\mathrm{i}}+1\right)}\right]}{\left[\left(q_{i} / p_{i}\right) e^{-\left(p_{i}+q_{i}\right)\left(t-\tau_{\mathrm{i}}+1\right)}+1\right]}=\frac{\left[1-e^{-(p+q)\left(t-\tau_{\mathrm{i}}+1\right)}\right]}{\left[(q / p) e^{-(p+q)\left(t-\tau_{\mathrm{i}}+1\right)}+1\right]}
$$

- Islam and Meade model

In this model, the parameters change across generations, and therefore $\mathrm{p}_{\mathrm{i}}$ and $\mathrm{q}_{\mathrm{i}}$ are different for all $\mathrm{i}$.

$$
F_{i}(t)=\frac{\left[1-e^{-\left(p_{i}+q_{i}\right)\left(t-\tau_{\mathrm{i}}+1\right)}\right]}{\left[\left(q_{i} / p_{i}\right) e^{-\left(p_{i}+q_{i}\right)\left(t-\tau_{\mathrm{i}}+1\right)}+1\right]}
$$

Step2. Test the constancy of parameters

This step discusses the constancy of parameters. Test the parameters to see whether they change across generations or not in order to determine to apply NB model or IM model, respectively. The processes are as follows:

1. Define Norton and Bass model as restricted model

Define Islam and Meade model as unrestricted model

2. Set up the hypothesis

$$
\begin{aligned}
& H_{0}: \Delta p_{i}=0 \text { and } \Delta q_{i}=0 \text { for all } i \\
& H_{1}: \text { At least one of } \Delta p_{i}, \Delta q_{i} \neq 0
\end{aligned}
$$

3. Test statistic

Compute the test statistic under the assumption that $\mathrm{H}_{0}$ is true. The W statistic follows a chi-square distribution as follows. $W=2(\log$ likelihood (unrestricted model)- 
$\log$ likelihood (restricted model)) $\sim \chi^{2}(v)$, where $v$ is the number of parameter restrictions.

This hypothesis can be tested by a likelihood ratio test or using full information maximum likelihood (FIML) estimation.

4. Compute sample test statistic

We can use the historical demand data to compute the sample test statistic. After computing the sample test statistic $\left(W_{0}\right)$, we can make a decision to reject the $H_{0}$ or we do not reject $H_{0}$.

\section{Test rules}

This step shows that how to make a decision rule to reject $H_{0}$ assumption or we do not reject $H_{0}$. The decision rules are as follows:

- If $W_{0}>\chi_{\alpha}^{2}(v)$, then we reject $H_{0}$. We have sufficient evidence to prove that $\mathrm{p}_{\mathrm{i}}$ and $\mathrm{q}_{\mathrm{i}}$ change across generations.

- If $W_{0}<\chi_{\alpha}^{2}(v)$, then we don't reject $H_{0}$. We do not have sufficient evidence to prove that $\mathrm{p}_{\mathrm{i}}$ and $\mathrm{q}_{\mathrm{i}}$ change across generations.

6. Conclusions

We can know $p_{i}$ and $q_{i}$ whether they change across generations or not via this test of hypothesis.

Step3. Incorporate price effects

Incorporate the diffusion model (Bass, Krishnan, and Jain 1994) into the proposed model in order to consider the price factor and therefore the expression of $F(t)$ in the Norton and Bass model are substituted for the expression of $F(t)$ in the model which Bass et al. suggested. The expressions in this proposed model are as follows.

$$
\begin{aligned}
& F_{i}(t)=\frac{\left[1-e^{-\left(X_{i}(t)-X_{i}(0)\right)\left(p_{i}+q_{i}\right)}\right]}{\left[(q / p) e^{-\left(X_{i}(t)-X_{i}(0)\right)\left(p_{i}+q_{i}\right)}+1\right]} \\
& X_{i}(t)=t+\ln \left(p r_{i}(t) / p r_{i}(0)\right) \beta
\end{aligned}
$$

Step4. Incorporate growth rates and seasonal factors

Growth rates and seasonal factors are significant factors for demand. Hence, it needs to incorporate these factors in the proposed model. In SMPRT model, we use multiplicative model to express the seasonal variation and growth rate effect. The method to express the seasonality and growth rate is to multiply the seasonal factor and market growth rate directly. The details are as follows.

1. The expression of the revised demand:

$$
\hat{s}_{i}(t) \times \alpha_{t} \times \exp \left(g_{t}\right)
$$

2. Prepare to estimate the parameters by using nonlinear least square method and revise the objective function as follows.

$$
\begin{aligned}
& \text { Original: } \operatorname{Min} \sum_{i=1}^{n} \sum_{t=1}^{l}\left[s_{i}(t)-\hat{s}_{i}(t)\right]^{2} \\
& \text { Revised: } \operatorname{Min} \sum_{i=1}^{n} \sum_{t=1}^{l}\left[s_{i}(t)-\hat{s}_{i}(t) \times \alpha_{t} \times \exp \left(g_{t}\right)\right]^{2}
\end{aligned}
$$

where $\alpha_{1}=1, \alpha_{t}=\alpha_{t-4}, \alpha_{t} \geq 0$

$$
g_{t}: \text { constant }
$$

Finally, SMPRT model integrates as follows:

$$
\begin{aligned}
& \operatorname{Min} \sum_{i=1}^{n} \sum_{t=1}^{l}\left[s_{i}(t)-\hat{s}_{i}(t) \times \alpha_{t} \times \exp \left(g_{t}\right)\right]^{2} \\
& \text { subject to } \\
& s_{i}(t)=f_{i}(t)\left[M_{i}+f_{i-1}(t)\left[M_{i-1}+f_{i-2}(t)\left[M_{i-2}+\cdots f_{2}(t)\left[M_{2}+f_{1}(t) M_{1}\right] \cdots\right]\right]\left[1-f_{i+1}(t)\right]\right. \\
& f_{i}(t)=F_{i}(t)-F_{i}(t-1) \\
& F_{i}(t)=\left\{\begin{array}{l}
\left(1-e^{-\left(X_{i}(t)-X_{i}(0)\right)\left(p_{i}+q_{i}\right)}\right) /\left(\left(q_{i} / p_{i}\right) e^{-\left(X_{i}(t)-X_{i}(0)\right)\left(p_{i}+q_{i}\right)}+1\right), t \geq \tau_{i} \\
0 \quad, t<\tau_{i}
\end{array}\right. \\
& X_{i}(t)=\left(t-\tau_{i}+1\right)+\ln \left(p r_{i}(t) / p r_{i}(0)\right) \beta \\
& \alpha_{1}=1, \alpha_{t}=\alpha_{t-4}, \alpha_{t} \geq 0 \\
& g_{t}: \operatorname{constant} \\
& 0<p_{i}<1 \\
& 0<q_{i}<1 \\
& M_{i}>0, \forall i=1,2, \cdots, n
\end{aligned}
$$

\subsection{Parameters Estimation}

There are several parameters in SMPRT model. This study estimates the parameters with nonlinear least square method. To estimate the parameters in SMPRT model is based on the historical demand data from the transaction database. In this approach, the parameters estimation can be divided into two stages.

Estimate the parameters in SMPRT model. In SMPRT model, the coefficient of innovation $\left(\mathrm{p}_{\mathrm{i}}\right)$, the coefficient of imitation $\left(\mathrm{q}_{\mathrm{i}}\right)$, the total market potential $\left(\mathrm{M}_{\mathrm{i}}\right)$, the seasonal factor $\left(\alpha_{t}\right)$, the effectiveness of the price ( $\beta$ ) must be estimated.

\subsection{Forecasting}

In the part of forecast, the data will be divided into two groups: training data and testing data. Training data are used to estimate the parameters in SMPRT model and subsequently the forecasting demand can be predict by the values of parameters in SMPRT model. However, testing data are used to validate the results of the forecasting demand and the actual demand.

\subsection{Results Interpretation and Evaluation}

The useful information or patterns can be extracted from the proposed demand forecast framework. The product life cycle and product demand forecast may help firms to plan their strategies for uncertainty and risk situations in the future. If the proposed demand forecast framework fails to forecast demand, it will lead the idle capacity or lose the orders. The result of demand forecast can be interpreted and analyzed in the discussions with domain experts to find the best model to solve the problem. 


\section{Chien, Chen, and Peng}

This study flows through the research framework. After estimating the parameters in the diffusion model, the statistical analysis will show the results of the estimated values. The coefficient of determination $\left(R^{2}\right)$ represents the explanation ability of the model according to the result of statistical analysis. The higher $R^{2}$ represents the diffusion model that has higher explanation ability for explaining the demand of semiconductor products. Though the result of statistical analysis, the values of the estimated parameters can be tested by p-value. Test the values of the estimated parameters whether they can be accepted or not.

The complete diffusion model can be obtained after getting the value of the parameters. Through the diffusion model, we can describe the patterns of the product life cycle and forecast product demand.

Forecasting error is a criterion to evaluate forecasting performance. In this study, it uses MAPE to measure the forecasting performance of the proposed model. The following is the formula of MAPE:

$$
M A P E=\frac{1}{S} \sum_{j=1}^{S}\left|\frac{Y_{j}-\hat{Y}_{j}}{Y_{j}}\right| \times 100 \%
$$

where $Y_{j}$ : actual value, $\hat{Y}_{j}$ : estimated value and $S$ : the number of samples.

The value of MAPE represents the forecasting performance. The smaller MAPE represents a higher performance for forecasting the product demand in the future.

\section{EMPIRICAL STUDY}

An empirical case study in semiconductor industry is conducted to evaluate and validate the demand forecast framework in this chapter.

\subsection{Problem Definition}

Newer technologies continually develop nowadays. Newer technologies are more complexity and high level than older one due to shorten the line-width and the utilization of a wafer. While newer technologies have less information to forecast demand, the difficulty and complexity are increased to predict the demand of newer technologies.

The quantity of semiconductor products affects the capacity allocation and capacity level. This study is applied to long-term and short-term capacity planning. Capacity allocation for the quantity of different semiconductor products under each technology belongs to short-term capacity planning. On the other hand, capacity expansion for the total quantity of different semiconductor products under each technology belongs to long-term capacity planning.

The proposed diffusion model is conducted to validate by using the real case study in semiconductor industry. This study constructed a systematic framework to handle with the demand forecasting problems. It hopes to describe the PLC and predict the demand of semiconductor products precisely for well capacity allocation and capacity expansion that maximum the utilization and profits by using the constructed demand framework in section 2 .

\subsection{Data Preparation}

The historical transaction data are collected by quarters and stored in the database. Before proceeding with the demand forecast framework, it needs to extract historical demand data from the database and then conduct the data preparation including data collection, cleaning, partition and integration.

Since the real data are proprietary information, the real data need to transform to protect the company. Without loss of generality, the real data are transformed for this empirical study. This study aimed to predict the semiconductor demand precisely for well capacity planning. Semiconductor products contain various products. This study selects a semiconductor product to conduct a validation. This semiconductor product $\mathrm{X}$, which is a leading product, is adopted in this study.

The time scale of this empirical case study is quarter. The total time horizon for the data of the semiconductor

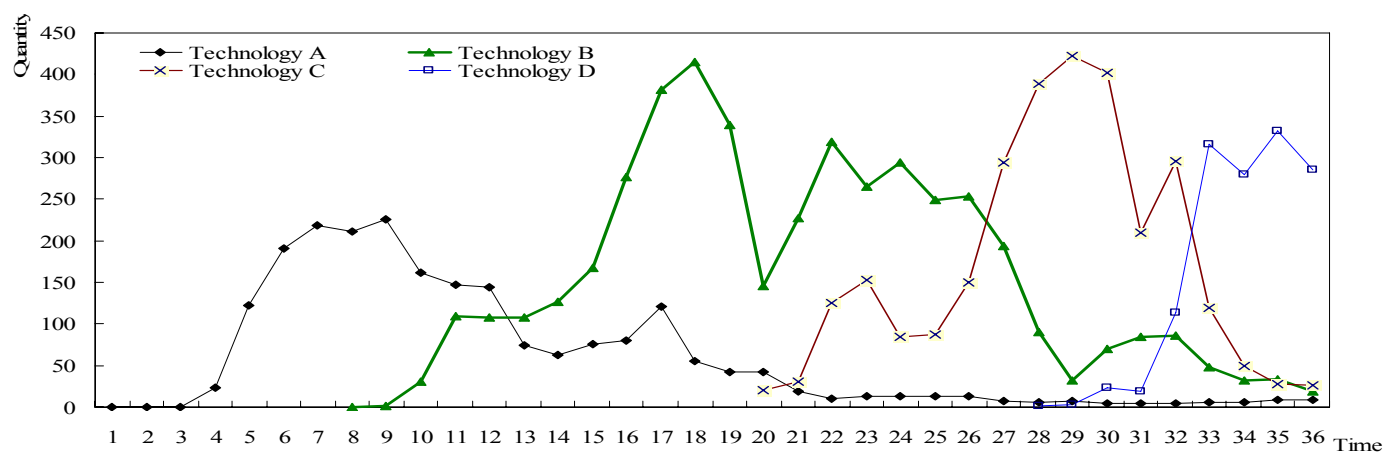

Figure 2: Semiconductor product demand under different technologies 


\section{Chien, Chen, and Peng}

product $\mathrm{X}$ is 36 quarters. The semiconductor product $\mathrm{X}$ is manufactured under different technologies. Additionally, the data of four generations of this semiconductor product $X$ cover 36, 29, 17 and 9 quarters.

The data are collected from the transaction database. After collecting the data, it has to conduct data cleaning, data partition, and data integration. The requisite data in the proposed diffusion model includes the price of the product, the growth rate of the semiconductor industry and the quantity of the product demand and therefore it have to get rid of the unnecessary data for this proposed model. Furthermore, integrate the requisite data in the proposed diffusion model into a worksheet in order to conduct a validation conveniently. Finally, sum up the quantity of the product $\mathrm{X}$ under the same technology in the same quarter.

The data consists of historical demand semiconductor product $\mathrm{X}$ under different technologies, unit price (dollar/per transistor), and sales growth rate (\%). Sales growth rate of quarterly financial reports $(\%)=($ net sales of current quarter - net sales of the previous quarter)/ net sales of the previous quarter. Figure 2 shows the historical demand semiconductor product $\mathrm{X}$ under each technology.

\subsection{Diffusion Model Construction}

Semiconductor product $\mathrm{X}$ has four generations and the total time horizon for the data is 36 quarters. We use the quantity of demand to replace the sales. Additionally, the introduction time of four generations of this semiconductor product $\mathrm{X}$ denotes 1st, 8th, 20th, 28th quarters respectively corresponding the time relative to generation 1 starting at $\mathrm{t}=1$.

Step1. Expand NB / IM model with four generation

$$
\begin{aligned}
& s_{1}(t)=f_{1}(t) M_{1}\left[1-f_{2}(t)\right] \\
& s_{2}(t)=f_{2}(t)\left[M_{2}+M_{1} f_{1}(t)\right]\left[1-f_{3}(t)\right] \\
& s_{3}(t)=f_{3}(t)\left[M_{3}+f_{2}(t)\left[M_{2}+M_{1} f_{1}(t)\right]\right]\left[1-f_{4}(t)\right] \\
& s_{4}(t)=f_{4}(t)\left[M_{4}+f_{3}(t)\left[M_{3}+f_{2}(t)\left[M_{2}+f_{1}(t) M_{1}\right]\right]\right]
\end{aligned}
$$

where

$$
\begin{aligned}
& f_{i}(t)=F_{i}(t)-F_{i}(t-1) \\
& F_{i}(t)=\left\{\begin{array}{cc}
\frac{\left[1-e^{-\left(p_{i}+q_{i}\right)\left(t-\tau_{i}+1\right)}\right]}{\left[\left(q_{i} / p_{i}\right) e^{-\left(p_{i}+q_{i}\right)\left(t-\tau_{i}+1\right)}+1\right]}, & t \geq \tau_{i} \\
0 & , t<\tau_{i}
\end{array}\right. \\
& \tau_{1}=1, \tau_{2}=8, \tau_{3}=20, \tau_{4}=28
\end{aligned}
$$

$\underline{\text { Step2. }}$ Test the constancy of parameters

(1) $H_{0}: \Delta p_{i}=0$ and $\Delta q_{i}=0$ for all $i, i=2,3,4$

$H_{1}:$ At least one of $\Delta p_{i}, \Delta q_{i} \neq 0, i=2,3,4$

(2) Get the values of log likelihood for unrestricted and restricted by using the FIML estimation in SAS. The expressions are as follows:

$W_{0}=2(-701.0875-(-749.3858))=96.5966$
(3) Because $\chi_{0.05}^{2}(4)=9.488<W_{0}=96.5966$, we have sufficient evidence to prove that $p_{i}$ and $q_{i}$ change across different generations.

We can know that $p_{i}$ and $q_{i}$ in this empirical case study change across different generations via this test.

Step3. The proposed model for this empirical case study The expressions for the semiconductor product $\mathrm{X}$ of this empirical case study are as follows:

$\operatorname{Min} \sum_{i=1}^{4} \sum_{t=1}^{36}\left[s_{i}(t)-\hat{s}_{i}(t) \times \alpha_{t} \times \exp \left(g_{t}\right)\right]^{2}$

subject to

$$
\begin{aligned}
& s_{1}(t)=M_{1} f_{1}(t)\left[1-f_{2}(t)\right] \\
& s_{2}(t)=f_{2}(t)\left[M_{2}+M_{1} f_{1}(t)\right]\left[1-f_{3}(t)\right] \\
& s_{3}(t)=f_{3}(t)\left[M_{3}+f_{2}(t)\left[M_{2}+M_{1} f_{1}(t)\right]\right]\left[1-f_{4}(t)\right] \\
& s_{4}(t)=f_{4}(t)\left[M_{4}+f_{3}(t)\left[M_{3}+f_{2}(t)\left[M_{2}+f_{1}(t) M_{1}\right]\right]\right] \\
& f_{i}(t)=F_{i}(t)-F_{i}(t-1) \\
& F_{1}(t)= \begin{cases}\left(1-e^{-\left(X_{1}(t)-X_{1}(0)\right)\left(p_{1}+q_{1}\right)}\right) /\left(\left(q_{1} / p_{1}\right) e^{-\left(X_{1}(t)-X_{1}(0)\right)\left(p_{1}+q_{1}\right)}+1\right) & , t \geq 1 \\
0 & , t<1\end{cases} \\
& F_{2}(t)= \begin{cases}\left(1-e^{-\left(X_{2}(t)-X_{2}(0)\right)\left(p_{2}+q_{2}\right)}\right) /\left(\left(q_{2} / p_{2}\right) e^{-\left(X_{2}(t)-X_{2}(0)\right)\left(p_{2}+q_{2}\right)}+1\right) & , t \geq 8 \\
0 & , t<8\end{cases} \\
& F_{3}(t)= \begin{cases}\left(1-e^{-\left(X_{3}(t)-X_{3}(0)\right)\left(p_{3}+q_{3}\right)}\right) /\left(\left(q_{3} / p_{3}\right) e^{-\left(X_{3}(t)-X_{3}(0)\right)\left(p_{3}+q_{3}\right)}+1\right) & , t \geq 20 \\
0 & , t<20\end{cases} \\
& F_{4}(t)= \begin{cases}\left(1-e^{-\left(X_{4}(t)-X_{4}(0)\right)\left(p_{4}+q_{4}\right)}\right) /\left(\left(q_{4} / p_{4}\right) e^{-\left(X_{4}(t)-X_{4}(0)\right)\left(p_{4}+q_{4}\right)}+1\right) & , t \geq 28 \\
0 & , t<28\end{cases} \\
& X_{1}(t)=(t-1+1)+\ln \left(p r_{1}(t) / p r_{1}(0)\right) \beta \\
& X_{2}(t)=(t-8+1)+\ln \left(p r_{2}(t) / p r_{2}(0)\right) \beta \\
& X_{3}(t)=(t-20+1)+\ln \left(p r_{3}(t) / p r_{3}(0)\right) \beta \\
& X_{4}(t)=(t-28+1)+\ln \left(p r_{4}(t) / p r_{4}(0)\right) \beta \\
& \alpha_{1}=1, \alpha_{\mathrm{t}}=\alpha_{\mathrm{t}-4}, \alpha_{t} \geq 0 \\
& g_{t}: \text { constant } \\
& 0<p_{i}<1 \\
& 0<q_{i}<1 \\
& M_{i}>0, \forall i=1,2,3,4
\end{aligned}
$$

\subsection{Estimation}

This study used nonlinear least squares procedure SYSNLIN in SAS to estimate the parameters in SMPRT model.

The values of estimated parameters are significance due to their p-values are smaller than 0.05 . The parameter estimates show that innovative coefficients $\left(\mathrm{p}_{1}-\mathrm{p}_{4}\right)$ are smaller than imitative coefficients $\left(\mathrm{q}_{1}-\mathrm{q}_{4}\right)$ in each technology. Additionally, the parameter estimates of total market potential $\left(\mathrm{M}_{1}-\mathrm{M}_{4}\right)$ are positive which are expected. The effectiveness of price $(\beta)$ is negative. If the price decreases, the sales will increase. Seasonal factors show that the first quarter is larger than other quarters. 


\subsection{Forecasting}

The data will be divided into two groups: training data and testing data. There are 36 data in this empirical case study. We use 34 quarters' data prior to estimate the parameters in the proposed multi-generation diffusion model and forecast the quantity of the product demand for prospective 2 quarters. After forecasting the product demand for prospective 2 periods, compare the forecasted results with the actual data.

\subsection{Results Interpretation and Evaluation}

Table 2: Estimated results of different models

\begin{tabular}{lc}
\hline & $\begin{array}{c}\text { Average } \\
\text { adjusted R }\end{array}$ \\
\hline $\begin{array}{l}\text { Speece and MacLachlan model } \\
\text { (without growth rate and seasonal factor) }\end{array}$ & $87.6 \%$ \\
\hline $\begin{array}{l}\text { SMPRT model } \\
\text { (without growth rate and seasonal factor) }\end{array}$ & $92.7 \%$ \\
\hline $\begin{array}{l}\text { Speece and MacLachlan model } \\
\text { (with growth rate and seasonal factor) }\end{array}$ & $88.3 \%$ \\
\hline $\begin{array}{l}\text { SMPRT model } \\
\text { (with growth rate and seasonal factor) }\end{array}$ & $94.8 \%$ \\
\hline
\end{tabular}

Table 3: The forecasting results for all technologies

\begin{tabular}{ccc}
\hline & $\begin{array}{c}\text { MAPE } \\
\text { (for 1Q-ahead) }\end{array}$ & $\begin{array}{c}\text { MAPE } \\
\text { (for 2Q-ahead) }\end{array}$ \\
\hline Technology A & $97.1 \%$ & $97.6 \%$ \\
Technology B & $4.6 \%$ & $6.35 \%$ \\
Technology C & $4.6 \%$ & $15.6 \%$ \\
Technology D & $0.1 \%$ & $10.9 \%$ \\
\hline Overall & $2.0 \%$ & $10 \%$ \\
\hline
\end{tabular}

In this section, we summarize the results of estimation and forecast.

Speece and MacLachlan (1992) also incorporated the price factor into multi-generation model for milk package type. SMPRT model is compared with Speece and MacLachlan model for semiconductor product $\mathrm{X}$ with the results summarized in Table 2.

SMPRT consists of market growth rate and seasonal factor is a good fit than other models from Table 2. Figure 3 shows the results of predicted and actual demand for all technologies.

Table 3 shows the forecasting results for 1-ahead and 2-head of all technologies with MAPE. SMPRT model has a precision forecast in one period except for technology A. As the forecasting periods expand, the forecasting performances are down. The forecasting results of technology A are not good. It can find that the demand of technology A fluctuates in the last periods. Because technology $\mathrm{A}$ is the older technology, some categories might have been maintained to produce by technology A. As a result of the situation, SMPRT model doesn't have good performances in technology A. In general, the forecasting performances are reasonable.

\section{CONCLUSION}

This study constructed a systematic method to forecast semiconductor products demand and therefore proposed a multi-generation diffusion model with considering the technological substitution, repeating purchases, price, growth rate, and seasonal factors for semiconductor products in order to improve the inherent forecasting methods are mainly designed for a single product or a single technology. An empirical study is conducted to validate the proposed SMPRT model with real data of semiconductor products. The results have shown a good fit and precise forecast for the semiconductor products. This approach can provide valuable information for managers to make their decisions.

Furthermore, there are other factors in other diffu-

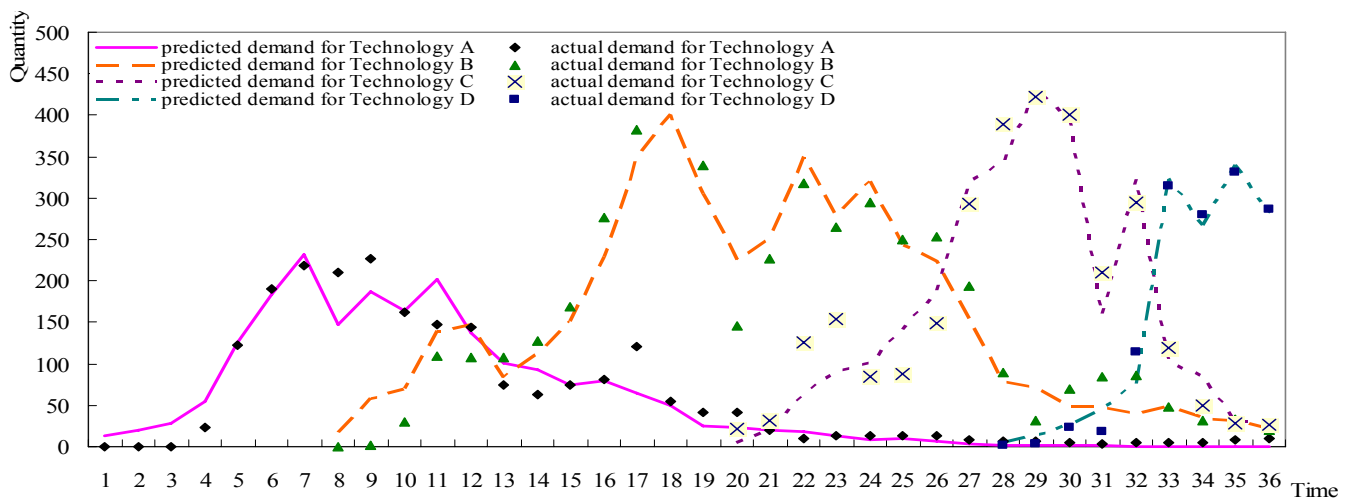

Figure 3: Results of SMPRT model for all technologies 


\section{Chien, Chen, and Peng}

sion models can be investigated in future studies to examine its appropriateness of incorporating them into the proposed model. Also, some assumptions in the proposed SMPRT model that need further investigations continually in future research. Thus, more situations can be considered into the multi-generation diffusion model in order to expand the multi-generation diffusion model effectively.

\section{ACKNOWLEDGEMENTS}

This research is supported by National Science Council (NSC 96-2628-E-007 -035 -MY3) and the Faulty Empowerment Award of National Tsing Hua University from the funding of the Ministry of Education in Taiwan.

\section{REFERENCES}

Bass, F. M. 1969. A new product growth model for consumer durables. Management Science 15:215-227.

Bass, F. M., T. V. Krishnan, and D. C. Jain. 1994. Why the Bass model fits without decision variables. Marketing Science 13:203-223.

Chien, C.-F., H. Wang, and M. Wang. 2007. A UNISON Framework for Analyzing Alternative Strategies of IC Final Testing for Enhancing Overall Operational Effectiveness. International Journal of Production Economics 107:20-30.

Islam, T., and N. Meade. 1997. The diffusion of successive generation of a technology: A more general model. Technological Forecasting and Social Change 56:49-60.

Kurawarwala, A. A., and H. Matsuo. 1998. Product growth models for medium-term forecasting of short life cycle products. Technological Forecasting and Social Change 57:169-196.

Leachman, R., S. Ding, and C.-F. Chien. 2007. Economic Efficiency Analysis of Wafer Fabrication. IEEE Transactions on Automation Science and Engineering 4(4): 501-512.

Mahajan, V., E. Muller, and F. M. Bass. 1990. New product diffusion models in marketing: A review and directions for research. Journal of Marketing 54:1-26.

Meade, N., and T. Islam. 2006. Modeling and forecasting the diffusion of innovation - A 25-year review. International Journal of Forecasting 22:519-545.

Norton, J. A., and F. M. Bass. 1987. A diffusion theory model of adoption and substitution for successive generations of high-technology products. Management Science 33:1069-1086.

Norton, J. A., and F. M. Bass. 1992. Evolution of technological generations: The law of capture. Sloan Management Review 33:66-77.

Rogers, E. M. 2003. Diffusion of Innovations. 5th ed. New York: Free Press.
SAS Institute Inc. 2004. SAS/ETS 9.1 user's guide. SAS Institute, Inc.

Speece, M. W., and D. L. Maclachlan. 1992. Forecasting fluid milk package type with a multigeneration new product model. IEEE Transactions on Engineering Management 39:169-175.

Speece, M. W., and D. L. Maclachlan. 1995. Application of a multi-generation diffusion model to milk container technology. Technological Forecasting and Social Change 49:281-295.

Srinivasan, V., and C. H. Mason. 1986. Nonlinear least squares estimation of new product diffusion models. Marketing Science 5:169-178.

Wu, J.-Z., and C.-F. Chien. 2008. Modeling strategic semiconductor assembly outsourcing decisions and an empirical study. OR Spectrum 30: 401-430.

\section{AUTHOR BIOGRAPHIES}

CHEN-FU CHIEN is a Professor in the Department of Industrial Engineering and Engineering Management and EMBA, National Tsing Hua University (NTHU). Since 2005, he has been on-leave to serve as the Deputy Director of Industrial Engineering Division in Taiwan Semiconductor Manufacturing Company. He received B.S. degree with double majors (with Phi Tao Phi Honor) in Industrial Engineering and Electrical Engineering from NTHU in 1990. He received M.S. in Industrial Engineering and Ph.D. of Operations Research and Decision Sciences from the University of Wisconsin-Madison in 1994 and 1996, respectively. He was a Fulbright Scholar in the Department of Industrial Engineering and Operations Research, UC Berkeley from 2002 to 2003 and received Executive Training in Harvard Business School in 2007. Dr. Chien is a Steering Committee Member of Industrial Engineering Division in National Science Council, Taiwan. $\mathrm{He}$ is Board Member of the Chinese Institute of Industrial Engineers (CIIE) and Chinese Institute of Decision Sciences. He is an Associate Editor of IEEE Transactions on Automation Science and Engineering and an Advisory Board Member of OR Spectrum. His research and development efforts focus on decision analysis, modeling and analysis for semiconductor manufacturing, dada mining, and manufacturing strategy. He received Outstanding Research Award and Tier 1 Principal Investigator (Top 3\%) from National Science Council, Distinguished UniversityIndustry Collaborative Research Award from Ministry of Education, Distinguished Young Industrial Engineer Award, Distinguished Young Faculty Research Award from NTHU, Best Paper Award from CIIE, Best Engineering Paper Award by Chinese Institute of Engineers, Taiwan.

YUN-JU CHEN received M.S. in Industrial Engineering and Engineering Management at the National Tsing Hua 
University. Her research interests include demand forecast and statistical method.

JIN-TANG PENG is an Assistant Professor in the Department of Business Administration, Yuanpei University. He received the M.S. and Ph.D. degrees in Industrial Engineering and Electrical Engineering from NTHU in 1998 and 2004, respectively. His research interests include demand forecast, data mining and rough set theory. 